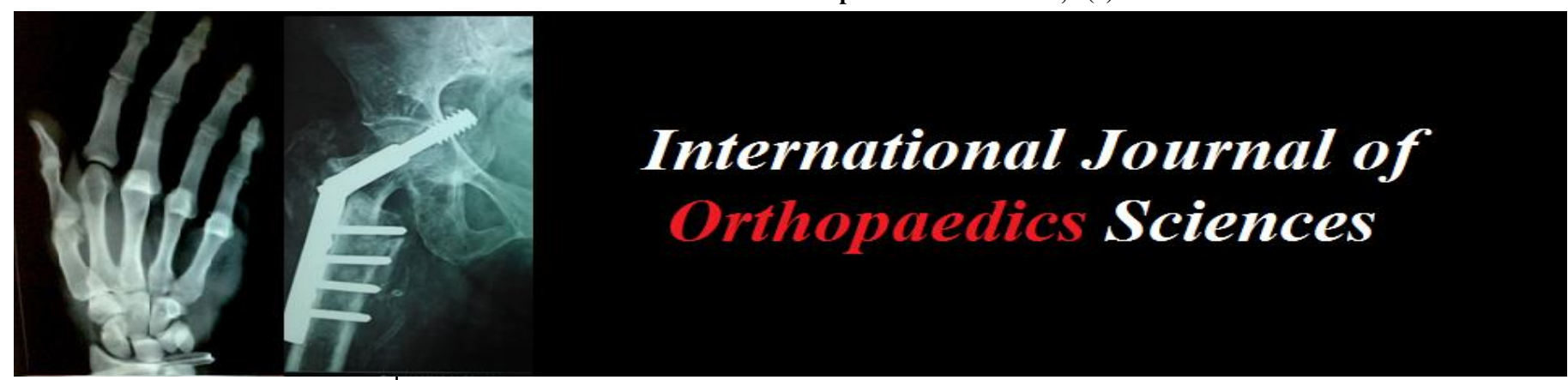

E-ISSN: 2395-1958

P-ISSN: 2706-6630

IJOS 2020; 6(1): 663-667

(C) 2020 IJOS

www.orthopaper.com

Received: 09-11-2019

Accepted: 13-12-2019

\section{Sandeep Kalia}

Associate Professor, Department

of Orthopedics, Dr. Rajendra

Prasad Government Medical

College, Tanda, Kangra,

Himachal Pradesh, India

\section{Narvir Singh Chauhan}

Associate professor, Department of Radiodiagnosis, Dr. Rajendra Prasad Government Medical

College- Tanda, District-Kangra, Himachal Pradesh, India

\section{Dinesh Sood}

Professor and Head, Department of Radiodiagnosis, Dr. Rajendra Prasad Government Medical College, Tanda, Kangra,

Himachal Pradesh, India

Indrajeet Singh Chauhan Junior Resident, Department of Radiodiagnosis, Dr. Rajendra Prasad Government Medical

College, Tanda, Kangra,

Himachal Pradesh, India

\section{Preeti Takkar}

Senior Resident, Department of

Radiodiagnosis, Dr. Rajendra

Prasad Government Medical

College, Tanda, Kangra,

Himachal Pradesh, India

Corresponding Author:

Dr. Narvir Singh Chauhan Dr. Rajendra Prasad

Government Medical College,

Tanda Campus, Kangra,

Himachal Pradesh, India

\section{MDCT evaluation of fractures injuries of joints in patients with lower extremity trauma: A single institution prospective observational study}

\author{
Sandeep Kalia, Narvir Singh Chauhan, Dinesh Sood, Indrajeet Singh \\ Chauhan and Preeti Takkar
}

DOI: https://doi.org/10.22271/ortho.2020.v6.i11.1940

\section{Abstract}

Background: Lower extremity fracture is a disabling injury which requires accurate and detailed characterization for optimal management. Conventional radiographic evaluation is hampered by under estimation of extent and severity of fracture injury. In contrast, MDCT is much more accurate tool for evaluation of these injuries involving anatomically complex locations. Our study examines the role of MDCT in analyzing the fracture injuries of lower extremity joints.

Materials and Methods: It is a prospective cross-sectional observational study conducted after obtaining institutional ethics committee approval. A total of 123 patients of fracture injuries involving lower extremity joints were included in study. MDCT was performed in all patients fulfilling the inclusion and exclusion criteria. Detailed evaluation of fracture injuries was done on advanced CT workstation and institutional PACS system. Multiplanar reconstructions (MPR) and Volume rendering was done in each case for detailed evaluation.

Results: The mean age of the study participants was 44.7 yrs \pm 18.0 with Male: Female ratio of $2.44: 1$. Road side accident (RSA) was commonest mode of injury $(60.2 \%)$ followed by fall from height $(34.1 \%)$ \& assault $(5.6 \%)$. When the mode of injury was RSA, the commonest fracture in hip was posterior column (43\%); in knee- Schatzker type VI (30\%); in ankle - Talar fracture (44.4\%) while in fall from height, intertrochanteric fracture occurred most frequently in hip (19.6\%), Schatzker type II in knee $(24 \%)$ and calcaneal fracture in ankle $(44.4 \%)$.

Conclusion: MDCT is important diagnostic tool in evaluation of fracture injuries of lower extremity joints and MPR along with Volume Rendered 3D models are extremely helpful for better spatial characterization of these injuries. In these injuries the site of fracture and injury patterns observed in a particular joint show marked variance depending on the underlying mechanism and mode of injury.

Keywords: Fractures, lower limb, trauma, MDCT, joint injury

\section{Introduction}

In the extremity trauma, radiography is often the initial radiological investigation undertaken. It is however found to underestimate the extent and severity of injury, especially in complex areas such as the acetabulum, knee, ankle and a high percentage of fractures may go unrecognized due to the complex anatomy and sometimes subtle injuries. Radiography also frequently misjudges the degree of displacement of fracture fragments in the extremities. MDCT overcomes the shortcoming of radiographs and is extremely helpful in fracture detection, evaluation, characterization, and treatment planning ${ }^{[1,4]}$. Its usage has witnessed exponential growth in recent years and it has supplanted radiography as the most common modality for diagnostic evaluation and treatment guidance of complex or indistinct fractures. The MPR images give excellent structural detail, and the 3-D images help in understanding the spatial relationship, which is useful for fracture classification and surgical planning ${ }^{[5,7]}$. MDCT is also helpful in the follow-up of postoperative results, even in the presence of metallic implants. Tendon injuries can also be evaluated with MDCT.

In fractures involving acetabulum which has complex bony anatomy, acquisition of axial images followed by MPR in coronal and sagittal planes combined with 3-D imaging is quite helpful. 
Suspected occult fractures, atypical fractures, and complex triplane fractures Can be accurately detailed from imaging with MDCT. While the volume-rendered 3-D images give excellent overview of the trauma with clear anatomical landmarks for the surgeon, the MPR images give detailed bony information about the fractures. Classification is often changed to a more severe fracture type after CT with MPR and 3-D reformatting which has implications for the choice of treatment and for the surgical approach ${ }^{[8,10]}$.

Additional benefits of CT are that the quality of images is not compromised by the presence of a plaster cast and an injured patient can be imaged in any position due to its isotropic properties and high-quality images can still be obtained ${ }^{[11]}$.

\section{Materials and Methods}

Study Design: The present study was carried out in Departments of Orthopaedics \& Radiodiagnosis of Dr. Rajendra Prasad Government Medical College, Tanda (H.P). It is a prospective observational cross-sectional study of oneyear duration (July 2018 -July 2019) done after obtaining approval of institutional ethics committee (Registration no ECR/490/Inst/HP/2013; Approval no 61-811/2018). This study was performed on patients who had sustained lower limb extremities fractures involving joints and in whom further characterization by MDCT was warranted for treatment purpose. A total of 123 such patients fulfilling the inclusion and exclusion criteria were included in the study after obtaining a written informed consent.

Inclusion criteria: Patients with history of traumatic insult to the appendicular skeleton and who had sustained fractures involving lower limb joints (Hip; Knee or Ankle joint) and required $\mathrm{CT}$ scan for better delineation and characterization of the injury for treatment purposes. Exclusion Criteria: Patients with pain due to other causes, such as systemic diseases involving the musculoskeletal system, tumorous conditions, or infectious diseases. Female patients with suspected or confirmed pregnancy

Procedure: Complete details regarding age, sex, complaints, duration of symptoms and mode of injury was obtained on a predesigned proforma. Physical examination of the patient was done and findings recorded. CT of the patient was done in appropriate position (Supine/Prone) whichever was most comfortable to and on a16 slice MDCT Philips machine (Brillance 16) without administering the contrast material. A scout image preceded the helical acquisition and field-of-view was adapted to the sectional area of each joint. The bone algorithm was used for image reconstruction. CT scans were assessed and compared for fracture characterization on advanced data acquisition system and operating console by two senior Orthopedician and Radiologist with 10 years of experience in traumatology each.

\section{Statistical analysis}

Difference in proportions were tested using chi square test. A $\mathrm{p}$ value $<0.05$ was considered statistically significant. Analysis of Variance (Anova) test was applied for comparison of means Data entry was done using MS Excel 2013 and data analysis was done using SPSS version 23.0.

\section{Results}

Maximum patients were seen in the age group of 41-50 years and least in the age group of 15-20 years. The age distribution in the study population across various age groups is detailed in Chart 1:

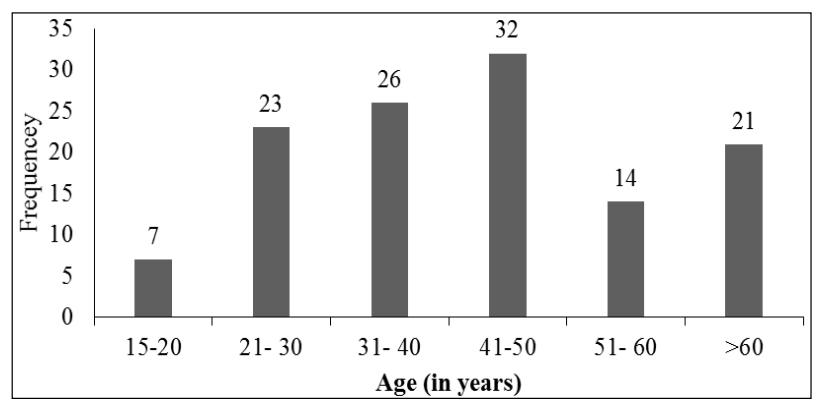

In our study, overwhelming majority of participants were male $(\mathrm{N}=87,71 \%)$, while females comprised only a minority of the study population $(\mathrm{N}=36,29 \%)$.

Mode of injury was analyzed and it was observed that road side accidents (RSA) were the commonest mode of injury, seen in 74 patients $(60.2 \%)$. The other modes of injury observed were- fall from height $(\mathrm{N}=42 ; 34.1 \%)$ and assault $(\mathrm{N}=7,5.7 \%)$ respectively. The difference among the groups was not statistically significant (p value 0.242 ).

Knee joint was overall the commonest joint to be involved in fracture injuries of lower limb $(\mathrm{N}=54 ; 43.9 \%)$ followed by Hip joint $(\mathrm{N}=51 ; 41.5 \%)$ and ankle joint $(\mathrm{N}=18 ; 14.6 \%)$ respectively. The difference among the groups was not statistically significant ( $p$ value 0.972 ).

Anatomical distribution pattern of fracture injuries were studied for different joints depending on mode of injury. The results for hip joint are summarized in Table 1; for Knee joint in Table 2 and for Ankle joint in Table 3.

Table 1: Distribution of study participants based on Mode of Injury \& type of fracture involving hip joint ( $\mathrm{n}=51)$

\begin{tabular}{|c|c|c|c|c|c|c|}
\hline \multirow{2}{*}{ Mode of injury } & \multicolumn{5}{|c|}{ Type of fracture } \\
\cline { 2 - 7 } & $\begin{array}{c}\text { Posterior } \\
\text { column }\end{array}$ & Anterior column & Inferior pubic ramus & Intertrochanteric & Anterior Wall & Greater trochanter \\
\hline Road side accident & $22(43 \%)$ & $14(27 \%)$ & $16(31 \%)$ & $1(2 \%)$ & $1(2 \%)$ & $1(2 \%)$ \\
\hline Fall from height & $2(4 \%)$ & $1(2 \%)$ & $2(4 \%)$ & $10(19.6 \%)$ & $1(2 \%)$ & $1(2 \%)$ \\
\hline Assault & 0 & 0 & $1(2 \%)$ & 0 & 0 & 0 \\
\hline Total & $24(47 \%)$ & $15(29.4 \%)$ & $19(37.2 \%)$ & $11(21.5 \%)$ & $2(4 \%)$ & $2(4 \%)$ \\
\hline
\end{tabular}

Table 2: Distribution of study participants based on Mode of Injury and type of fracture involving Knee joint (n=54)

\begin{tabular}{|c|c|c|c|c|c|c|c|c|c|c|c|}
\hline \multirow{3}{*}{ Mode of injury } & \multicolumn{11}{|c|}{ Type of fracture } \\
\hline & \multirow{2}{*}{$\begin{array}{c}\text { Lateral } \\
\text { Femoral } \\
\text { Condyle }\end{array}$} & \multirow{2}{*}{$\begin{array}{l}\text { Fibular } \\
\text { Head }\end{array}$} & \multirow{2}{*}{$\begin{array}{c}\text { Inter condylar } \\
\text { eminence }\end{array}$} & \multirow{2}{*}{$\begin{array}{l}\text { Medial Femoral } \\
\text { Condyle }\end{array}$} & \multirow{2}{*}{ Patella } & \multicolumn{6}{|c|}{ Schatzer Fracture Types } \\
\hline & & & & & & 1 & 2 & 3 & 4 & 5 & 6 \\
\hline Road side accident & $3(5.5 \%)$ & $17(31.4 \%)$ & $3(5.5 \%)$ & $3(5.5 \%)$ & $3(5.5 \%)$ & $1(1.8 \%)$ & 0 & $1(1.8 \%)$ & $5(9.2 \%)$ & $4(7.4 \%)$ & $14(30 \%)$ \\
\hline Fall from height & $3(5.5 \%)$ & $4(7.4 \%)$ & $1(1.8 \%)$ & $2(3.7 \%)$ & 0 & 0 & $13(24 \%)$ & $2(3.7 \%)$ & $1(1.8 \%)$ & 0 & 0 \\
\hline Assault & $1(1.8 \%)$ & $1(1.8 \%)$ & 0 & $3(5.5 \%)$ & 0 & 0 & 0 & 0 & 0 & 0 & 0 \\
\hline Total & $7(13 \%)$ & $22(40 \%)$ & $4(7.4 \%)$ & $8(14 \%)$ & $3(5.5 \%)$ & $1(1.8 \%)$ & $13(24 \%)$ & $3(5.5 \%)$ & $6(11 \%)$ & $4(7.4 \%)$ & $14(30 \%)$ \\
\hline
\end{tabular}


Table 3: Distribution of study participants based on Mode of Injury and type of fracture involving ankle joint $(\mathrm{n}=18)$

\begin{tabular}{|c|c|c|c|}
\hline \multirow{2}{*}{ Mode of injury } & \multicolumn{3}{|c|}{ Type of fracture } \\
\cline { 2 - 4 } & Bi-malleolar & Talus & Calcaneus \\
\hline Road side accident & $4(22.2)$ & $8(44.4)$ & $5(27.7)$ \\
\hline Fall from height & $1(5.5)$ & $2(11)$ & $8(44.4)$ \\
\hline Assault & 0 & 0 & 0 \\
\hline Total & $5(27.7)$ & $10(55.5)$ & $13(72)$ \\
\hline
\end{tabular}

\section{Discussion}

CT is fast replacing radiography as the standard imaging modality for orthopaedic trauma because of several advantages it offers like: a) Ability to characterise the fracture in great detail; b) Identification of occult fractures which are not apparent on radiographs; c) Periosseous soft tissue information; d) Generation Clinician friendly 3 D reconstruction images ${ }^{[12]}$

The present study was undertaken to evaluate the utility of MDCT in characterization of lower extremity joint fractures affecting the Hip, Knee and Ankle joints as these joints have anatomically complex configuration and radiographs are likely to underestimate the severity and extent of injury.

The mean age of the patients in our study group was $44.7 \pm$ 18.01 years, the patients in the age group 41 to 50 years constituted the largest group $(\mathrm{N}=32)$ followed by 31to 40 $(\mathrm{N}=26)$. The least number of participants were from the age group of 15-20 years $(\mathrm{N}=7)$.

Out of 123 patients in our study, 87(71\%) were males \& $36(29 \%)$ were females (M:F is 2.44:1).

Mode of injury was analyzed in each participant and three distinct modes were observed. The most frequent mode of injury was found to be high velocity road side accidents involving motor vehicles. This mode was the underlying mechanism in $60.2 \%$ of observed injuries $(\mathrm{N}=74)$ in the study population. A probable explanation for this high occurrence is the hilly terrain of our state which results in greater number of RSA. The second important mode of injury observed was a fall from height which accounted for $34.1 \%$ of sustained injuries $(\mathrm{N}=42 \%)$. Assault constituted the smallest subset and only $5.6 \%$ patients $(\mathrm{N}=7)$ came under this category. Similar results were seen in a study done by Chan et al. ${ }^{[13]}$ which shows the impact of MDCT for fracture detection where majority of the fractures were due to high velocity vehicular injuries.

In terms of gender wise distribution of mode of injury, it was observed that in male gender, road side accidents was found to be the commonest mode of injury seen in $67.4 \% \quad(\mathrm{~N}=58)$ patients followed by fall from height seen in $24.3 \%(\mathrm{~N}=21)$ patients \& assault seen in $8.3 \%(\mathrm{~N}=7)$ patients. In females on the other hand majority had sustained injuries due to fall from height $56.7 \%(\mathrm{~N}=21)$, followed by road side accidents in $40.6 \%(\mathrm{~N}=15)$ while only 1 female patient $(\mathrm{N}=2.7 \%)$ patient was referred due to injury sustained from assault. A gender based variation in the mode of injury was hence observed in our study.

Out of 123 patients in our study, maximum number of study participants underwent CT of the knee joint (43.9\%) followed by Hip joint (41.5\%) and ankle joint (14.6\%).

Fracture analysis of hip joint injuries (Figures 1 and 2) revealed that overall, fracture injury of posterior column fracture was the most common injury in this region $(47 \%)$ followed by fracture of inferior pubic ramus (37.2\%), anterior column $(29.4 \%)$, intertrochanteric region $(21.5 \%)$, greater trochanter $(4 \%) \&$ anterior wall $(4 \%)$. Correlation of Hip fractures with the mode of injury was done. In road side accidents it was observed that posterior column fracture once again the most common injury (43\%) followed by fracture of inferior pubic ramus (31\%), anterior column (27\%), intertrochanteric region $(2 \%)$, greater trochanter $(2 \%)$ \& anterior wall $(2 \%)$ in the patients who sustained injury by this mode. On the other hand in patients sustaining injury after a fall from height, intertrochanteric fracture $(20 \%)$ was found to be most common injury followed by fracture of posterior column (4\%), inferior pubic ramus (4\%), anterior column $(2 \%)$, greater trochanter $(2 \%) \&$ anterior wall $(2 \%)$. Thus a distinct variability in injury pattern was observed in our study based on the mechanism of injury.

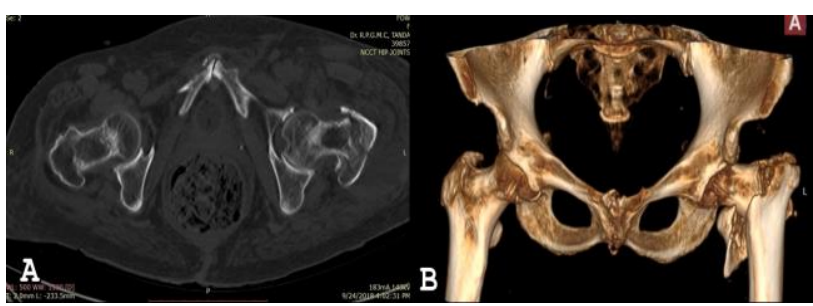

Fig 1: MDCT axial image (A) and Volume Rendered 3 D image (B) showing intertrochanteric fracture of left hip

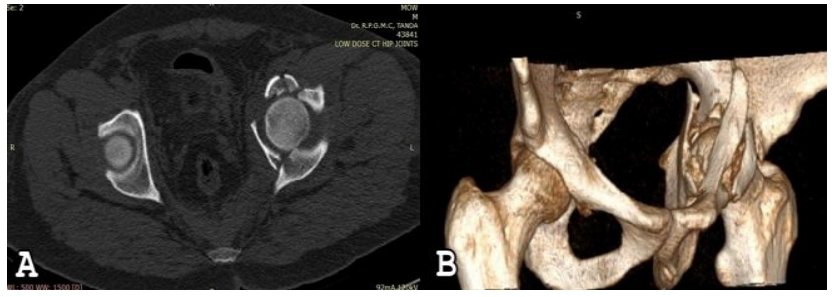

Fig 2: MDCT axial image (A) and Volume Rendered $3 \mathrm{D}$ image (B) showing fractures of anterior and posterior columns of left hip

In injuries of Knee joint, Tibial plateau fractures can show a wide range from subtle compression fractures to complex fractures that are difficult to visualize completely on radiography. MDCT is helpful in picking up these fractures and in delineating their full extent. It is also useful in assessing the articular surface involvement and evaluating additional periarticular soft tissue injuries, thus helping in better overall understanding of the injury.

Out of total 54 patients referred for knee joint related injuries (Figure 3), most common injury overall was found to be fracture of fibular head (40\%) which was followed by Schatzker type VI tibial plateau fracture (30\%), Schatzker type II tibial plateau fracture $(24 \%)$, medial femoral condyle fracture (14\%), lateral femoral condyle fracture(13\%), Schatzker type IV tibial plateau fracture (11\%), Schatzker type V tibial plateau fracture $(7.4 \%)$, intercondylar eminence fracture $(7.4 \%)$, Schatzker type III tibial plateau fracture (5.5\%), patellar fracture (5.5\%) \& Schatzker type I tibial plateau fracture $(1.8 \%)$. When the knee joint fracture injuries were analysed in terms of the mode of injury, it was found that in patients who sustained injuries due to road side accidents, the most common injury found was again fracture of fibular head(31.4\%) which was followed by Schatzker type VI tibial plateau fracture $(30 \%)$, Schatzker type IV tibial plateau fracture $(9.2 \%)$, Schatzker type V tibial plateau fracture $(7.4 \%)$, lateral femoral condyle fracture $(5.5 \%)$, patellar fracture $(5.5 \%)$, intercondylar eminence fracture $(5.5 \%)$, medial femoral condyle fracture $(5.5 \%)$, Schatzker type III tibial plateau fracture $(1.8 \%)$ \& Schatzker type I tibial plateau fracture $(1.8 \%)$

In the subset of patients who had sustained injuries after fall 
from height a very different injury pattern was observed. It was seen that Schatzker type II tibial plateau fracture (24\%) was found to be the most common injury followed by fibular head fracture $(7.4 \%)$, lateral femoral condyle fracture $(5.5 \%)$, Schatzker type III tibial plateau fracture $(3.7 \%)$, medial femoral condyle fracture $(3.7 \%)$, Schatzker type IV tibial plateau fracture $(1.8 \%) \&$ intercondylar eminence fracture $(1.8 \%)$. Similarly in cases of assault, medial femoral condyle was most commonly fractured $(5.5 \%)$, followed by fibular head fracture $(1.8 \%)$ \& lateral femoral condyle fracture $(1.8 \%)$.

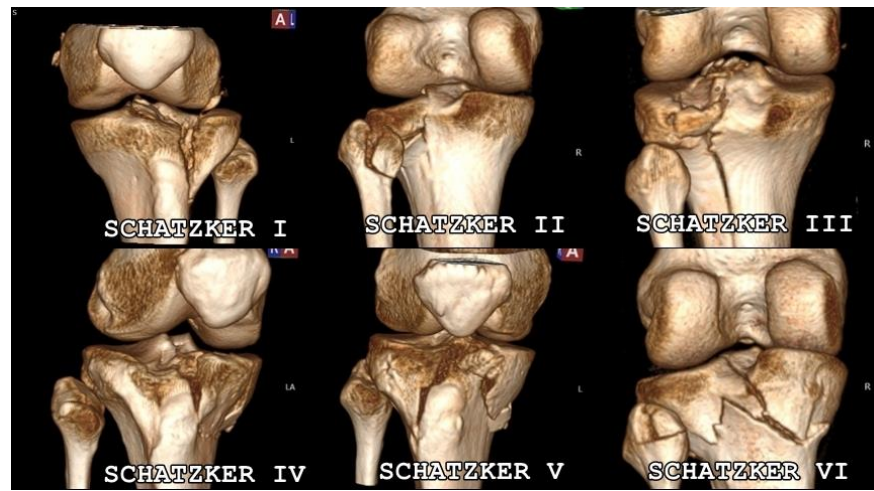

Fig 3: Volume rendered 3 D CT images showing the various types of Schatzker fractures of Knee

Similar results were observed in a prospective study by Wicky et al. ${ }^{[14]}$ in which out of 42 patients with knee injuries, where 18 patients were assigned a more severe classification after CT. In 13 cases, the surgical planning was altered based on the information gained from the $\mathrm{CT}$ studies.

MDCT results in significant improvement in the visualization of calcaneal fractures and fractures of the tibial plafond ${ }^{[15]}$. With MDCT, the degree of comminution is readily visible, and the relation between the fracture fragments, their size, and displacement is easier to appreciate than with radiography.

In patients scanned for the ankle joint related injuries, out of total 18 patients, most common injury found was calcaneum fracture $(72 \%)$ followed by talus fracture $(55.5 \%) \&$ bimalleolar fracture (27.7\%). In terms of mode of injury, after sustaining road side accidents most common injury found was talus fracture $(44.4 \%)$ followed by calcaneum fracture $(27.7 \%) \&$ bimalleolar fracture $(22.4 \%)$. In injuries sustained due to fall from height, most common injury found was a calcaneum fracture $(44.4 \%)$, followed by talus fracture $(11 \%)$ $\&$ bimalleolar fracture $(5.5 \%)$.

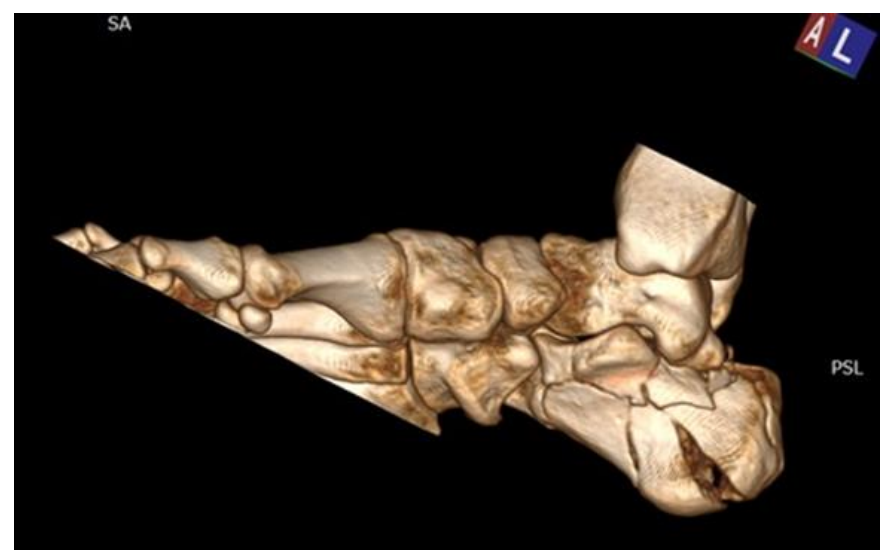

Fig 4: Volume rendered 3D CT image showing comminuted fracture of calcaneum

\section{Conclusions}

MDCT is an indispensable diagnostic imaging tool in evaluating the fracture injuries of lower extremity joints and should routinely be used in lower limb trauma protocols for better characterization of these complex injuries and also for treatment planning to ensure better outcome.

Pattern and site of fractures sustained differs according to the underlying mechanism and mode of injury. In high velocity trauma caused by road side accidents, the most common injury pattern in hip joint is posterior column fracture, in knee joint -Schatzker type VI fracture and in the ankle joint- talar fracture whereas in injuries sustained due to fall from height, intertrochanteric fracture is most common injury in hip joint, Schatzker type II fracture in in knee joint \& fracture calcaneum in the ankle joint.

\section{References}

1. Lubovsky O, Liebergall M, Mattan Y, Weil Y, Mosheiff R. Early diagnosis of occult hip fractures: MRI versus CT scan. Injury. 2005; 36:788-92.

2. Gill S, Smith J, Fox R, Chesser T. Investigation of occult hip fractures: the use of CT and MRI. The Scientific World Journal, 2013.

3. Markhardt BK, Gross JM, Monu J. Schatzker classification of tibial plateau fractures: use of CT and MR imaging improves assessment. Radiographics. 2009; 29:585-97.

4. Dodd A, Paolucci EO, Korley R. The effect of threedimensional computed tomography reconstructions on preoperative planning of tibial plateau fractures: a casecontrol series. BMC musculoskeletal disorders. 2015; 16:144.

5. Rydberg J, Buckwalter KA, Caldemeyer KS et al. Multisection CT: scanning techniques and clinical applications. Radiographics. 2000; 20:1787-806.

6. Geijer M, El-Khoury GY. MDCT in the evaluation of skeletal trauma: principles, protocols, and clinical applications. Emergency Radiology. 2006; 13:7-18.

7. Herzog C, Ahle H, Mack M et al. Traumatic injuries of the pelvis and thoracic and lumbar spine: does thin-slice multidetector-row CT increase diagnostic accuracy? European radiology. 2004; 14:1751-60.

8. Dahlen H, Franck W, Sabauri G, Amlang M, Zwipp H. Fehlklassifikation extraartikulärer distaler Radiusfrakturen in konventionellen Röntgenaufnahmen. Der Unfallchirurg. 2004; 107:491-8.

9. Wicky S, Blaser P, Blanc C, Leyvraz P, Schnyder P, Meuli R. Comparison between standard radiography and spiral CT with 3D reconstruction in the evaluation, classification and management of tibial plateau fractures. European radiology. 2000; 10:1227-32.

10. Chan PS, Klimkiewicz JJ, Luchetti WT et al. Impact of CT scan on treatment plan and fracture classification of tibial plateau fractures. Journal of orthopaedic trauma. 1997; 11:484-9.

11. Lee K. Global trends in maxillofacial fractures. Craniomaxillofacial trauma \& reconstruction. 2012; 5:213-22.

12. Konda S, Goch A, Leucht $\mathrm{P}$ et al. The use of ultra-lowdose CT scans for the evaluation of limb fractures: is the reduced effective dose using ct in orthopaedic injury (Reduction) protocol effective? The bone \& joint journal. 2016; 98:1668-73.

13. Chan PN, Antonio GE, Griffith JF, Yu KW, Rainer TH, Ahuja AT et al. Computed tomography for cervical spine 
trauma. The impact of MDCT on fracture detection and dose deposition. Emergency radiology. 2005; 11(5):28690.

14. Wicky S, Blaser P, Blanc C, Leyvraz P, Schnyder P, Meuli R et al. Comparison between standard radiography and spiral CT with 3D reconstruction in the evaluation, classification and management of tibial plateau fractures. European radiology. 2000; 10:1227-32.

15. Daftary A, Haims AH, Baumgaertner MR. Fractures of the calcaneus: a review with emphasis on CT. Radiographics. 2005; 25:1215-26. 